\title{
Pattern Recognition Neural Networks in Classification Groups of Partial Removable Dentures Lifetime
}

\author{
Abbas I. Hussein ${ }^{1 *}$, Imad H Abood ${ }^{2}$ and Waleed Bdaiwi Salih ${ }^{3}$ \\ ${ }^{1}$ College of Dentistry, University of Anbar, Iraq \\ ${ }^{2}$ College of Science, University of Anbar, Iraq \\ ${ }^{3}$ College of Education for PureScience, University of Anbar, Iraq
}

Received: November 07, 2013; Accepted: January 31, 2014; Published: February 4, 2014

*Corresponding author: Abbas I. Hussein, College of Dentistry, University of Anbar, Iraq, Tel: 00964-790-557-5987; E-mail: abbass_69@yahoo.com

\begin{abstract}
Classification is one of the most common research work associated with neural networks. This paper cast lighton the use of this technique as a robust statistical procedure for modeling data regarding problem of classification groups of partial removable dentures lifetime. Groups are either 1 which refers to lifetime exceeds average, and 0 which refers to lifetime less than or equal average lifetime. The main purpose of this work is to provide a new technique of classification as well as to stimulate further research interests and efforts in this topic.
\end{abstract}

Keywords: Lifetime (LT); Removable partial dentures lifetime (RPDLT); Pattern recognition neural networks (PRNN)

\section{Introduction}

Patients as well as dentists and technicians are aware about whether or not it is worthwhile spending money to enhance performance of partial dentures. This issue is actually very vague since it contains number of effected factors that plays significant roles in the performance of partial dentures. Most patients prefer to have partial dentures that lasts long time. Simply they do not like to pay money every once and again changing their partial dentures. Therefore, partial dentures lifetime is one of the essential factors that patients and dentists should pay attention about it.

In medical research works as in other scientific discipline classification procedures are very necessary in order to build allocation criteria so it can be easily to relate certain readings to a certain group and so on. The term pattern recognition can be defined as the task of assigning some item to a correct class of items based on the measurements about this item. Often, this task is tobe performed automatically with the aid of computer. Examples of the pattern recognition can be anything in real life. Accordingly, one may expect different pattern recognition tasks. In this contrast, one may conclude that pattern recognition is a system of classification.
Pattern recognition can also be defined as composite of features that are characteristic of an individual. In classification, a pattern is a pair of variables $\{\mathrm{x}, \mathrm{w}\}$ where $\mathrm{x}$ is a collection of observations or features (feature vector) and $w$ is the concept behind the observation (label). The quality of a feature vector is related to its ability to discriminate examples from different classes. Examples from the same class should have similar feature values and while examples from different classes having different feature values [1].

Neural networks have emerged as an important tool forclassification. The recent vast research activities in neuralclassification have established that neural networks are apromising alternative to various conventional classificationmethods. The advantage of neural networks lies in the followingtheoretical aspects. First, neural networks are datadriven self-adaptive methods in that they can adjust themselvesto the data without any explicit specification of functional ordistributional form for the underlying model. Second, they are universal functional approximators in that neural networks canapproximate any function with arbitrary accuracy $[2,3]$.

Matlab is a high-level language and interactive environment that enables the performing of computationally intensive tasks faster than with traditional programming languages such as $\mathrm{C}, \mathrm{C}++$, and FORTRAN. Accordingly, it has been proven that neural networks implemented in Matlab are faster than the ones implemented using the other programming languages. Studying their performance will help researchers use the best neural networks that fit their specific type of problem [4].

There are some common characteristics of all the types of neural networks in Matlab. First of all, the dataset should be prepared before it is given to a certain type of neural network. The data should consist of two parts: one input part that contains the input data and one target part that contains the classes in which the data should be organized by the networks, corresponding to the patterns in which the input data is classified. The target data should be represented as matrices, in which each row has 
a 1 in some position I, where I is the class they are to represent, and 0 's in the rest. Our classification contains only supervised neural networks, in which the learning is done knowing in which class an input should be placed, but we also experimented with unsupervised neural networks, which try to classify the input data based only on its characteristics, not using target data [5].

\section{Methods}

A sample of 150 patients who were visited private dental clinics in Ramadi city of AL-Anbar province-Iraq was considered in this study. Lifetime of RPD, age and residential area of patients were the main concerns to this study. Lifetime of RPD is actually the time since the RPD firstly used till the patient starts to experience problems with it. These variables consists the matrix $x$ that contained the vectors of the input set of the MATLAB PRNN.

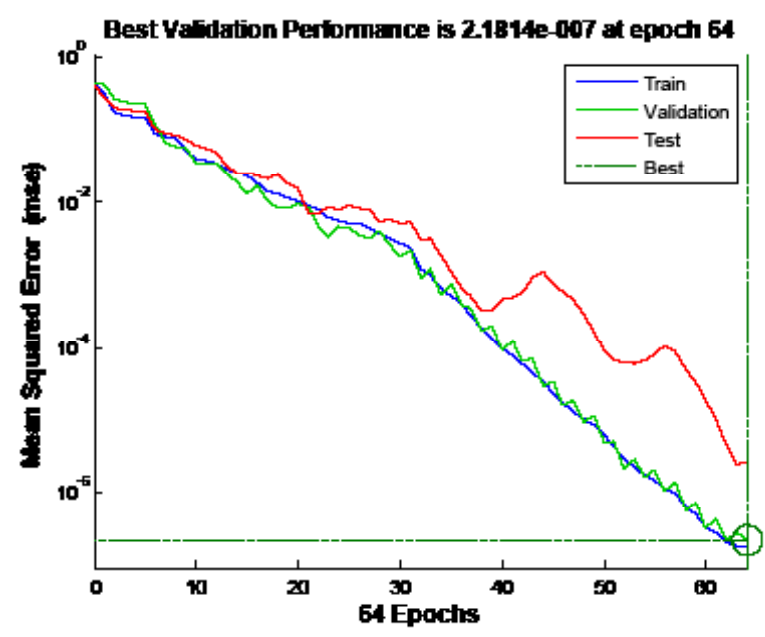

Figure 1: Best validation performance with regard to PRNN.
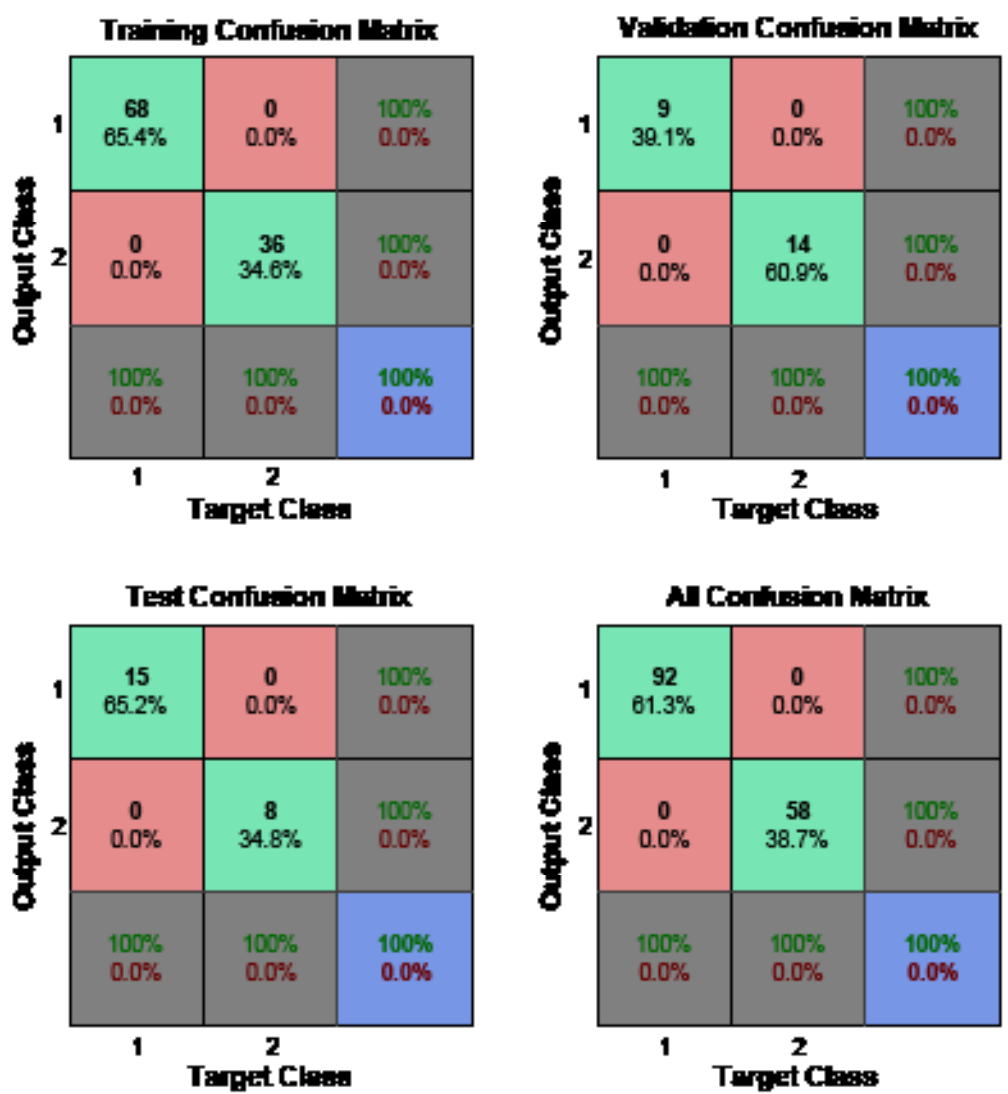

Figure 1: Best validation performance with regard to PRNN. 
The mean lifetime of approximately 50 months [6] was used as a criterion to code all those patients with RPD lifetime exceeds this mean as ones and all other as zeros. In this regard the target set of data will consist of two vectors, one vector for each group.

\section{Results}

The data that been collected for previous research work [6] was subdivided into two sets of data in order to facilitate for the requirements of the MATLAB pattern recognition neural networks. The first set is that of the input data which include three vectors; age, RPDLT, and residential area. On the other hand the target set was created according to the criteria imposed on the vector of the RPDLT. In this contrast two vectors were created each of them represent one group.

According to MATLAB results, figure 1 shows the best validation test which occur at epoch 64. Figure 2 shows the confusion matrix (classification matrix) of the data sets considered during the data analysis of MATLAB. One can easily noticed that this procedure is $100 \%$ classify all cases, whereas the linear discriminant function carried out by the use of STATISTICA 8.0 for the same set of data revealed eight wrong cases. This is of course makes the PRNN much better than that of the conventional techniques used in the cases of classification.

\section{Discussion}

Partial dentures are subject to many types of abuses whether that related to education level of patients, or that related to the environment where the patients live. With regard to the results of the linear discriminant function carried out by the STATISTICA 8.0, theresidential area of the patient play a significant role in determining the RPDLT. This is due to the essential differences between rural and urban life particularly in Arabic countries. Food habits and polluted environment are the most threatening factors affecting not only dental care but oral health generally.

Examining or changing RPD is a problem of many directions, one of the very important directions is that related to the economic status of the patients. In this context an estimates of the lifetime is very essential to patients to prepare to be ready for such dental procedure. Good lifetime estimate means good health plan and an encourage factor to keep patients within good health mode. According to the results of this paper it is very recommended not to continue wearing RPD after 50 months after the first use.

\section{Conclusion}

PRNN performed much better than conventional linear and quadratic discriminant functions. Data sets must be well prepared and the target matrix must contain vectors equals to the number of groups (classes). RPD should be subjected to a significant examination at month 50 after the first use in order to determine its validity.

\section{References}

1. http://research.cs.tamu.edu/prism/lectures/iss/iss_19.pdf

2. Cybenko G (1989) Approximation by superpositions of a sigmoidal function. Mathematics of Control, Signals and Systems 2(4): 303-314.

3. Devijver PA, Kittler J (1982) Pattern Recognition: A Statistical Approach. Prentice-Hall, Englewood Cliffs, New Jersey, USA.

4. http://www.mathworks.com/products/matlab

5. Haykin S (1994) Neural Networks: A comprehensive Foundation. Macmillan College Publishing, New York, USA.

6. Abass IH, Imad HA (2012) Estimation of partial dentures lifetime by the use of artificial neural networks. Egyptian Dental Journal 58(3). 